\title{
Las revistas científico-técnicas españolas de ciencias de la actividad física y el deporte: adecuación a las normas ISO y grado de normalización
}

\author{
José Devís Devís \\ Doutor en Ciencias de la Educación, Profesor de la Universidad de \\ Valencia. \\ E-mail: jose.devis@uv.es
}

Miguel Villamón Herrera

Licenciado en Educación Física, Profesor de la Universidad de Valencia.

E-mail:miguel.villamon@uv.es

\section{Luis Antolín Jimeno}

Doutor en Psicologia, Profesor de la Universidad de Valencia.

E-mail: luis.antolin@uv.es

Javier Valenciano Valcárcel

Licenciado en Educación Física, Becario de investigación.

E-mail: franva@alumni.uv.es

Alberto Moreno Doña

Licenciado en Educación Física, Profesor de la Carrera Pedagogía en Educación Física de la Universidad del Mar. La Serena. Chile.

E-mail:almodo@alumni.uv.es

\section{Resumen}

Este artículo tiene por objeto conocer el grado de normalización y la adecuación de las revistas científico-técnicas españolas del campo de las Ciencias de la Actividad Física y el Deporte a diversos apartados clave de las normas ISO 8-1977 e ISO 215-1986. A partir de la observación directa de los fascículos, se han confeccionado dos índices para calcular el Grado General de Normalización (GGN) y el Grado Fundamental de Normalización (GFN). Los resultados indican que el ajuste de las publicaciones a la norma ISO 8-1977 es equivalente a la de otros campos españoles como el de Economía y el de Tecnología, mientras que es menor en la norma ISO 215-1986. La mayoría de las revistas presentan un GFN inferior a su respectivo GGN. Como principal conclusión se señala la existencia de un margen considerable de mejora en las revistas estudiadas, especialmente en los aspectos

fundamentales de las normas.

\section{Palabras clave}

Normalización; Ciencias de la Actividad Física y el Deporte; Revistas españolas; Revistas científicas; Evaluación; Normas ISO.

Physical activity and sport sciences spanish scientific and technical journals: ISO norms adjustment and normalization grade

\section{Abstract}

The objective of this paper is to know the normalization grade and adjustment of Spanish scientific and technical journals of Physical Activity and Sport Sciences to various key epigraphs of the ISO 8-1977 and ISO 215-1986 norms. Also two indexes are made to calculate the General Normalization Grade (GGN) and the Fundamental Normalization Grade (GFN) of the journals. Results show that journals adjustment to ISO 8-1977 is equivalent to the journals of other Spanish fields as Economy and Technology, while it is less adjusted in relation to ISO 2151986. Most of the journals show an inferior GFN respective to its GGN. A considerable range of improvement in the journals studied, especially in the fundamental aspects of the ISO 215-1986 norm and some of the ISO 8-1977 norm, is the main conclusion of the study.

\section{Keywords}

Normalization; Physical Activity and Sport Sciences; Spanish journals; Scientific journals; Evaluation; ISO norms.

\section{INTRODUCCIÓN}

Con el crecimiento de la producción científica, experimentado desde mediados del siglo XX, surgió la necesidad de uniformar y estandarizar las publicaciones científicas para facilitar la comunicación del conocimiento científico y su difusión. Entre ellas destacan las revistas, debido al importante papel que desempeñan en la cadena de transmisión de la ciencia (Callon, Courtial y Penan, 1995). Así surgieron los distintos sistemas de normas para la publicación de revistas, tanto a nivel internacional como las ISO o a nivel nacional como las UNE en España y las ABNT en Brasil. Pero inmediatamente surgió la necesidad de comprobar que las normas que se iban creando las cumplían los editores de las revistas y, de esta manera, surgieron los estudios evaluativos de normalización. Detrás existía el interés por mejorar la calidad de los productos y servicios de documentación, evaluar la producción científica y orientar las políticas de subvención y ayuda a las revistas y la investigación en general. Desde entonces, la normalización de las publicaciones periódicas, entendida como el grado de adecuación o cumplimiento de algún sistema de normas, se ha revelado no sólo como un elemento eficaz de mejora de la comunicación, la difusión y la visibilidad, sino también como un factor clave en la producción, análisis y uso de las revistas científicas. Es decir, la normalización se ha convertido en un elemento de mejora de todo el sistema de transferencia de la información científica (Ruiz-Pérez y Pinto, 1990; Giménez, Román y Vázquez, 2001).

Los primeros estudios de normalización de las publicaciones periódicas provienen de la década de los años 60 y 70 del siglo pasado. Según Delgado (1997), se trata de estudios diversos que se han ocupado de las revistas de un área geográfica concreta, de una disciplina específica, de una institución editora o de una sola revista. Asimismo, la normalización también ha formado parte de las evaluaciones globales de la calidad de las revistas científicas, es decir, ha sido uno de sus elementos junto a otros relativos a la difusión y el contenido científico. En estos casos, el objetivo fundamental era ofrecer un modelo de evaluación que consiguiese aunar todos los factores de la calidad de las revistas para ofrecer criterios más racionales dirigidos a la asignación de fondos por parte de las agencias de recursos de investigación y a los 
Las revistas científico-técnicas españolas de ciencias de la actividad física y el deporte: adecuación a las normas ISO y grado de normalización

responsables de los sistemas de información (p.e. Krzyzanowski y Ferreira, 1998) (Suter, Román y UNAM, 1999)*.

En las evaluaciones globales, la importancia concedida a la normalización es variable dentro del conjunto de indicadores o criterios utilizados. En cualquier caso, esta dimensión se convierte en prioritaria y básica cuando no se conoce la situación en que se encuentran las revistas en un determinado campo del saber como es el de las Ciencias de la Actividad Física y el Deporte. Por ello, el presente artículo se orienta específicamente a la normalización de las publicaciones periódicas españolas de este campo. Si bien este trabajo forma parte de un estudio más amplio de análisis de la calidad de las revistas (ver Devís et al., 2003), el objetivo del mismo consiste en ofrecer una primera aproximación a la situación formal de dichas publicaciones, principales órganos de difusión y comunicación del conocimiento certificado. Desde nuestro punto de vista, conocer la adecuación a las normas ISO y el grado de normalización de las revistas científico-técnicas de las Ciencias de la Actividad Física y el Deporte es un primer paso en el proceso de mejora de la calidad de las revistas de un campo con una corta tradición académica y científica, al menos dentro del contexto español.

\section{METODOLOGÍA}

La estrategia metodológica empleada para la realización del estudio de normalización de las publicaciones periódicas españolas de carácter científico-técnico del mencionado campo de estudio se especifica en los siguientes cuatro apartados.

\section{Revistas sometidas a evaluación}

El análisis de las revistas de Ciencias de la Actividad Física y el Deporte exigía partir del directorio de las

* SUTER, T., ROMÁN, A; UNAM. Criterios para la inclusión de publicaciones periódicas en el catálogo Latindex. Documento sin publicar (gentileza de A. Román). Caracas, 1999.

TABLA 1
Revistas científico-técnicas de ciencias de la actividad física y el deporte

\begin{tabular}{|c|c|c|}
\hline $\mathrm{N}$ & Título y, en su caso, subtitulo & Editor \\
\hline 1 & $\begin{array}{l}\text { Agua y Gestión. Seae-info Revista especializada } \\
\text { en actividades acuáticas y gestión de } \\
\text { instalaciones deportivas. }\end{array}$ & SEAE (Esplugues de Llobregat, Barcelona) \\
\hline 2 & Apunts. Educación Física y Deportes & Generalitat de Catalunya. INEF-C (Barcelona) \\
\hline 3 & Apunts. Medicina del Deporte & Santiago Siquier Fadó (Esplugues de Llobregat, Barcelona) \\
\hline 4 & Archivos de Medicina del Deporte & Jon Ajuría Blanco (Pamplona) \\
\hline 5 & Áskesis & Editorial Pila Teleña (Madrid) \\
\hline 6 & $\begin{array}{l}\text { Avances en Traumatología, Cirugía, } \\
\text { Rehabilitación, } \\
\end{array}$ & Asepeyo (Barcelona) \\
\hline 7 & Biomecánica & Sociedad Ibérica de Biomecánica y Biomateriales (Barcelona) \\
\hline 8 & Cairon. Revista de Ciencias de la Danza & $\begin{array}{l}\text { Universidad de Alcalá de Henares (Alcalá de Henares, } \\
\text { Madrid) }\end{array}$ \\
\hline 9 & $\begin{array}{l}\text { Comunicaciones Técnicas. Publicación de la } \\
\text { Escuela Nacional de Entrenadores de la Real } \\
\text { Federación Española de Natación. }\end{array}$ & $\begin{array}{l}\text { Escuela Nacional de Entrenadores de la Real Federación } \\
\text { Española de Natación (Madrid) }\end{array}$ \\
\hline 10 & Espacio y Tiempo. Revista de Educación Física & Asociación de Profesores de Educación Física (Almeria) \\
\hline 11 & Fútbol. Cuadernos Técnicos & Editorial Wanceulen (Sevilla) \\
\hline 12 & $\begin{array}{l}\text { Habilidad Motriz. Revista de las Ciencias de la } \\
\text { Actividad Física y el Deporte }\end{array}$ & $\begin{array}{l}\text { Ilustre Colegio Oficial de Licenciados en Educación Física y } \\
\text { en Ciencias de la Actividad Física y del Deporte de } \\
\text { Andalucía (Córdoba) }\end{array}$ \\
\hline 13 & $\begin{array}{l}\text { Infocoes. Publicación técnico-cientifica del } \\
\text { Centro Olimpico de Estudios Superiores }\end{array}$ & Centro Olímpico de Estudios Superiores \\
\hline 14 & $\begin{array}{l}\text { Motricidad. Revista Euro-Americana de Ciencias } \\
\text { de la Actividad Fisica y del Deporte }\end{array}$ & $\begin{array}{l}\text { Facultad de Ciencias de la Actividad Fisica y del Deporte. } \\
\text { Universidad de Granada (Granada) }\end{array}$ \\
\hline 15 & $\begin{array}{l}\text { Natación, Saltos/Sincro y Waterpolo. Revista de } \\
\text { la Asociación Española de Técnicos de } \\
\text { Natación. }\end{array}$ & Asociación Española de Técnicos de Natación (Lleida) \\
\hline 16 & Perspectivas de la Actividad Física y el Deporte & Junta de Castilla y León (León) \\
\hline 17 & \begin{tabular}{|l|}
$\begin{array}{l}\text { Psicomotricidad. Revista de estudios y } \\
\text { experiencias }\end{array}$ \\
\end{tabular} & Instituto de Psicomotricidad (Madrid) \\
\hline 18 & Revista de Biomecánica & Instituto de Biomecánica de Valencia (Paterna, Valencia) \\
\hline 19 & $\begin{array}{l}\text { Revista de Educación Física. Renovar la Teoría y } \\
\text { la Práctica }\end{array}$ & Boidecanto (La Coruña) \\
\hline 20 & Revista de Entrenamiento Deportivo & Boidecanto (La Coruña) \\
\hline 21 & Revista de Psicología del Deporte & Universitat de les Illes Balears (Palma de Mallorca) \\
\hline 22 & Revista española de Educación Física y Deportes & $\begin{array}{l}\text { Consejo General de Colegios Oficiales de Licenciados en } \\
\text { Educación Fisica y en Ciencias de la Actividad Física y del } \\
\text { Deporte (Madrid) }\end{array}$ \\
\hline 23 & $\begin{array}{l}\text { Revista Internacional de Medicina y Ciencias de } \\
\text { la Actividad Física y del Deporte (on-line) }\end{array}$ & Vicente Martínez de Haro (Madrid) \\
\hline 24 & Revista Juridica del Deporte & Aranzadi Editorial (El Cano, Navarra) \\
\hline 25 & $\begin{array}{l}\text { Selección. Revista española e iberoamericana de } \\
\text { Medicina de la Educación Fisica y el Deporte }\end{array}$ & $\begin{array}{l}\text { Federación Española de Especialistas en Medicina de la } \\
\text { Educación Física y el Deporte (Madrid) }\end{array}$ \\
\hline 26 & Tándem. Didáctica de la Educación Física & Editorial Graó (Barcelona) \\
\hline
\end{tabular}

publicaciones periódicas científico-técnicas que serían sometidas a evaluación. Ante la ausencia de un índice o directorio específico de tales revistas, el equipo investigador tuvo que elaborar un listado propio mediante un proceso estructurado en varias fases.

Si bien el proceso se ha descrito con más detalle en otro lugar (Devís, et al., 2003), las fases consistieron en: 1) establecimiento de un primer listado de revistas deportivas españolas a partir de la consulta de varios directorios y bases de datos; 2) listado resultante de seleccionar las revistas de la fase anterior que disponían de ISSN, estaban activas en el año 2000 y no eran boletines de información ni revistas de divulgación o periodísticas; y 3) listado definitivo después de validar el anterior con entrevistas a expertos de las distintas disciplinas del campo. De esta manera se obtuvieron las 26 revistas que se recogen en la tabla 1 . 


\section{Criterios de normalización para el análisis}

Los criterios o indicadores de normalización, objeto del presente trabajo, fueron extraídos de las normas ISO 8 1977 referidas a la 'Presentación de las publicaciones periódicas' y las normas ISO 215-1986 referidas a la 'Presentación de artículos en publicaciones periódicas' (ISO, 1988), equivalentes a las normas UNE 50-101-90 y UNE 50-133-94. En concreto, de la norma ISO 8-1977 se consideraron los siguientes aspectos, recogidos de forma abreviada en los apartados siguientes:

- Apartado 3: Título de la publicación periódica

3.1. Que sea corto y fácil de citar.

3.2. Que sea acorde a la temática que trata la revista.

3.4. Su uniformidad. Que se escriba de la misma manera en la portada, en el sumario o índice y en la primera página del texto (ésta última interpretada como la primera después de la segunda página de cubierta que no sea la que incluye el sumario ni una página de publicidad).

- Apartado 4: Fascículos

4.1. Que mantengan el mismo formato.

4.2. Que incluyan en la tapa los datos que siguen: título, número de volumen (en su caso), número de fascículo, ISSN y fecha de publicación en números arábigos.

\section{- Apartado 5: Numeración}

5.1. Que los fascículos mantengan numeración correlativa dentro de un volumen (si lo hubiera), comenzando por 1 el primero de cada volumen (en el caso de que no tenga volumen también deben ser correlativos).

- Apartado 8: Presentación

8.1. Que los distintos fascículos de una publicación mantengan la uniformidad tipográfica.

8.2. Que los artículos (incluidos los de opinión) de una misma publicación mantengan la uniformidad tipográfica.

- Apartado 9: Título de cada página: Que todas las páginas incluyan las siguientes indicaciones: título de la publicación (o abreviado), fecha, número de volumen (si lo hubiera), fascículo, número de página, título del artículo (o abreviado), nombre de autores o primer autor.
- Apartado 10: Paginación

10.1. Que la paginación de un volumen sea correlativa, es decir, que englobe todos los fascículos y no comience de nuevo en cada uno de ellos. Esta paginación empleará solamente cifras arábigas.

10.3. Que las páginas de publicidad o anuncios estén numeradas aparte y puedan suprimirse fácilmente al encuadernar la revista.

- Apartado 12: Sumario del fascículo

12.1. Que se halle en la primera página a continuación de la segunda de cubierta.

12.2. Que indique para cada artículo: nombre del autor o autores, título completo y número de la primera y última página.

12.4. Que especifique, tras los artículos, posibles secciones como 'noticias' o 'congresos' y que estén separadas por una interlínea.

De la misma forma, de la norma ISO 215-1986, referida a la presentación de artículos en publicaciones periódicas, se consideraron los criterios que detallamos abreviadamente:

- Apartado 4: Elementos de identificación

4.1.1. Que aparezca el título de manera destacada.

4.2.1. Que figure el nombre y apellidos del autor o de los autores. Si son más de uno pueden abreviarse.

4.3.1. Que se adjunte un resumen del artículo en el idioma de la revista y en inglés o francés.

4.3.2. Que aparezcan palabras clave.

- Apartado 5: Texto principal

5.8. Que se detalle la bibliografía referenciada (en nuestro caso que aparezca la bibliografía).

\section{Procedimiento de evaluación}

La evaluación de las revistas se realizó mediante la observación directa del último ejemplar del año 2000 de cada una de las revistas del listado definitivo. Por exigencia de algunos indicadores se observó el resto de números del mismo año o de años anteriores en el caso de las revistas de periodicidad anual. La perspectiva de análisis adoptada consistió en el cumplimiento o grado de cumplimiento de los aspectos normativos seleccionados para este estudio. 
Las revistas científico-técnicas españolas de ciencias de la actividad física y el deporte:

adecuación a las normas ISO y grado de normalización

Para proporcionar una visión global y comparativa del grado de normalización de las revistas, se emplearon unos índices matemáticos similares a los elaborados en estudios españoles anteriores (Delgado y Ruiz, 1998; Redero, 2002), simplificados y adaptados a nuestro trabajo. Estos índices son el Grado General de Normalización (GGN) y Grado Fundamental de Normalización (GFN). El GGN integra, para su cálculo, todos los criterios o indicadores recogidos de la ISO 81977 y de la ISO 215-1986. Es decir, un máximo de 35 criterios que pueden disminuir si la revista carece de alguno de los criterios a evaluar. El GFN, por su parte, recoge únicamente aquellos criterios de las normas que han sido considerados como fundamentales. Es decir, un máximo de 20 criterios que pueden ser menos si no existen determinados criterios en algunas revistas. De la norma ISO 8-1977 se han considerado fundamentales los 5 criterios relativos a los datos de la tapa o cubierta (apartado 4.2.), los 7 relativos a los datos de cada página (apartado 10.1.), y los 4 primeros relativos al sumario de cada fascículo (apartado 13.1. y 13.2.). De la norma ISO 215-1986 se ha considerado fundamental la identificación de los autores, la existencia de resumen en el idioma de la revista y en inglés o francés (apartado 4.2.1. y 4.3.1.), así como la existencia de referencias bibliográficas (apartado 5.8.).

Los dos índices se calculan conforme a las siguientes fórmulas:

1) Índice del Grado General de Normalización: GGN = $\mathrm{NeG} / \mathrm{Nt}$

- NeG: el número de criterios que se cumplen total o parcialmente $^{*}, \mathrm{y}$

- Nt: 35 o el número total de criterios de las normas UNE 101-50-90 y UNE 50-133-94 posee una determinada revista en particular

2) Índice del Grado Fundamental de Normalización: $\mathrm{GFN}=\mathrm{NeF} / \mathrm{Ntf}$

- NeF: el número de criterios fundamentales que se cumplen total o parcialmente ${ }^{*}, \mathrm{y}$

- Ntf: el número total de criterios fundamentales para una determinada revista.

\footnotetext{
* Como algunos elementos se analizaron de acuerdo a una escala de cumplimiento (Nunca; Casi nunca; Casi siempre; Siempre), se han asignado los valores siguientes a cada uno de esos niveles de la escala para efectuar los cálculos de los grados de normalización: Nunca $=0$; Casi nunca $=0.25$; Casi siempre $=0.75 ;$ y Siempre $=1$.
}

TABLA 2

Escala de normalización para el GGN y el GFN

\begin{tabular}{|c|c|}
\hline Nivel de la Escala & Franja \\
\hline Muy Elevado & Entre 0,85 y 1 \\
\hline Elevado & Entre 0,70 y 0,84 \\
\hline Medio & Entre 0,50 y 0,69 \\
\hline Bajo & Menos de 0,50 \\
\hline
\end{tabular}

Finalmente, para completar la interpretación de los datos resultantes y poder tener una idea más precisa de la comparación de las revistas se ha considerado una escala de normalización que establece cuatro niveles en los grados del GGN y del GFN, según puede observarse en la tabla 2.

\section{Instrumentos de recogida de datos}

Los diversos instrumentos de recogida de datos que se han utilizado en esta investigación son de tres tipos: a) fichas de las revistas científico-técnicas; b) listas de comprobación para el análisis; y c) cuestionario para expertos.

Las fichas de las revistas tienen por objeto recoger información general de cada una de las publicaciones periódicas del listado definitivo. Las listas de comprobación sirven para recoger información de los criterios o indicadores de las normas ISO 8-1977 e ISO 215-1986, referida respectivamente a la 'Presentación de las publicaciones periódicas' y a la 'Presentación de artículos en publicaciones periódicas'. El cuestionario para expertos sirve para validar el segundo listado del inventario de revistas y poder obtener el definitivo, ya que a los 25 expertos se les pidió, entre otras cosas, que añadieran otras revistas además de las que contenía el listado del mencionado cuestionario.

\section{RESULTADOS}

\section{Presentación de las publicaciones periódicas (ISO 8-1977)}

El análisis de las revistas en lo referente al título completo indica que se ajusta a la temática en el 100\% de los casos. Los títulos son cortos y fáciles de citar en el 92,3\% de las revistas (no incluye los subtítulos), quedando fuera de este porcentaje dos publicaciones, 'Revista Internacional de Medicina y Ciencias de la Actividad Física y el Deporte' y la denominada 'Avances en Traumatología, Cirugía, Rehabilitación, Medicina Preventiva y Deportiva' (tabla 3, a seguir). 
La uniformidad del título en la portada, el sumario y la primera página de texto se cumple 'siempre' en algo más de la mitad de las revistas $(53,8 \%)$ y no se cumple 'nunca' solamente en la revista 'Fútbol. Cuadernos Técnicos'.

En cuanto al formato y otros datos identificativos (ver tabla 4), 'Biomecánica' es la única revista que no mantiene el mismo formato entre los fascículos del mismo volumen o año del conjunto de las 26 publicaciones periódicas del campo. El título y el número de volumen y de fascículo (si tienen) está presente en la cubierta de todas las revistas, mientras que el ISSN sólo aparece en la cubierta del 19,2\% de las revistas. Es destacable que 'Motricidad' e 'Infocoes' posean ISBN, el código de identificación propio de los libros, cuando el que les corresponde a las publicaciones periódicas es el ISSN.
TABLA 3

Titulo de las publicaciones periódicas

\begin{tabular}{|c|c|c|c|c|c|c|c|c|}
\cline { 2 - 9 } \multicolumn{1}{c|}{} & \multicolumn{2}{|c|}{ Acorde a temática } & \multicolumn{2}{c|}{ Corto y fácil citar } & \multicolumn{4}{c|}{ Uniformidad } \\
\cline { 2 - 9 } \multicolumn{1}{c|}{ Sí } & No & Sí & No & Nunca & Casi nunca & $\begin{array}{c}\text { Casi } \\
\text { siempre }\end{array}$ & Siempre \\
\hline $\mathrm{N}^{\circ}$ & 26 & 0 & 24 & 2 & 1 & 1 & 10 & 14 \\
\hline$\%$ & 100 & 0 & 92,3 & 7,7 & 3,8 & 3,8 & 38,5 & 53,8 \\
\hline
\end{tabular}

\section{TABLA 4}

Formato, datos identificativos y numeración de los fascículos

\begin{tabular}{|c|c|c|c|c|c|c|c|c|c|c|c|c|c|c|c|c|c|}
\hline & \multirow{2}{*}{\multicolumn{2}{|c|}{ Igual forma }} & \multicolumn{13}{|c|}{ Datos en tapa } & \multirow{2}{*}{\multicolumn{2}{|c|}{$\begin{array}{l}\text { Número } \\
\text { correlativo }\end{array}$}} \\
\hline & & & \multicolumn{2}{|c|}{ Título } & \multicolumn{3}{|c|}{$\mathrm{N}^{\circ} \mathrm{vol}$} & \multicolumn{3}{|c|}{ No fasc. } & \multicolumn{3}{|c|}{ ISSN } & \multicolumn{2}{|c|}{ Fecha } & & \\
\hline & Sí & No & Sí & No & Sí & No & $\mathrm{Nt}$ & Sí & No & $\mathrm{Nt}$ & Sí & No & $\mathrm{Nt}$ & Sí & No & Sí & No \\
\hline $\mathrm{N}^{\circ}$ & 25 & 1 & 26 & 0 & 14 & 0 & 12 & 25 & 0 & 1 & 5 & 19 & 2 & 24 & 2 & 20 & 6 \\
\hline$\%$ & 96,2 & 3,8 & 100 & 0 & 53,8 & 0 & 46,2 & 96,2 & 0 & 3,8 & 19,2 & \begin{tabular}{|l}
73,1 \\
\end{tabular} & 7,7 & 92,3 & 7,7 & 76,9 & 23,1 \\
\hline
\end{tabular}

\section{TABLA 5}

\section{Presentación tipográfica}

\begin{tabular}{|c|c|c|c|c|c|c|}
\hline & \multicolumn{6}{|c|}{ Uniformidad tipográfica } \\
\hline & \multicolumn{4}{|c|}{ De los artículos } & \multicolumn{2}{|c|}{ De los fascículos } \\
\hline & Nunca & Casi nunca & Casi siempre & Siempre & Sí & No \\
\hline $\mathrm{N}^{\circ}$ & 0 & 3 & 4 & 19 & 23 & 3 \\
\hline$\%$ & 0 & 11,5 & 15,4 & 73,1 & 88,5 & 11,5 \\
\hline
\end{tabular}

TABLA 6

Leyenda de cada página

\begin{tabular}{|c|c|c|c|c|c|c|c|c|c|c|c|c|c|c|c|c|c|}
\hline & \multicolumn{2}{|c|}{$\begin{array}{c}\text { Nombre } \\
\text { revista }\end{array}$} & \multicolumn{2}{|c|}{ Fecha } & \multicolumn{3}{|c|}{ Volumen } & \multicolumn{3}{|c|}{ Fascículo } & \multicolumn{3}{|c|}{$\mathrm{N}^{\circ}$ página } & \multicolumn{2}{|c|}{$\begin{array}{c}\text { Título } \\
\text { artículo }\end{array}$} & \multicolumn{2}{|c|}{$\begin{array}{c}\text { Nombre } \\
\text { autores }\end{array}$} \\
\hline & Sí & No & Sí & No & Sí & No & $\mathrm{N}$ & S1 & No & $\mathrm{Nt}$ & Sí & $\mathrm{Nc}$ & $\mathrm{N}$ & Si & No & Sí & No \\
\hline $\mathrm{N}^{\circ}$ & 17 & 9 & 9 & 17 & 6 & 8 & 12 & 14 & 11 & 1 & 24 & 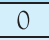 & 7 & 5 & 21 & 5 & 21 \\
\hline$\%$ & 65,4 & 34,6 & 34,6 & 65,4 & 23,1 & 30,8 & 46,1 & 53,9 & 42,3 & 3,8 & 92,3 & 0 & 7,7 & 19,2 & 80,8 & 19,2 & 80,8 \\
\hline
\end{tabular}

Por otra parte, la numeración correlativa de los fascículos se respeta en el $76,9 \%$ de los casos, mientras que el resto de las revistas no numera los fascículos de un nuevo volumen desde el número 1. En la revista 'Psicomotricidad' parece confundirse el volumen con el fascículo, es decir, se denomina volumen a lo que debería ser el fascículo y viceversa. De hecho, si se intercambiaran las denominaciones la numeración de los fascículos sería correlativa y, por tanto, correcta. En el caso de 'Comunicaciones Técnicas', la numeración no es correlativa porque indica el 'año 2000' como si fuera el volumen y por eso comienzan cada año a numerar los fascículos por el número 1.

El análisis de la presentación (ver tabla 5) indica que las únicas revistas que 'nunca' emplean la misma tipografía entre los fascículos del mismo volumen o año (el 11,5\%) son la 'Revista Española de Educación
Física y Deportes', 'Áskesis' y la 'Revista Internacional de Medicina y Ciencias de la Actividad Física y el Deporte'. Además, estas mismas revistas 'casi nunca' mantienen la uniformidad tipográfica de los artículos, mientras que el $73,1 \%$ de las publicaciones lo hacen 'siempre' y el 15,4\% 'casi siempre'.

La leyenda de cada página consta de diversas indicaciones, destacando por su completo cumplimiento el número de página, a excepción de las dos revistas on line que no tienen, lo que corresponde con el 92,3\% de las revistas. El nombre de la revista se cumple en el 65,4\% de las revistas, el número del fascículo en el $53,9 \%$ y el volumen en el 23,1\%, aunque el 46,1\% no tiene volumen. El menor cumplimiento se presenta en el título del artículo y el nombre de los autores, ya que sólo son recogidos por el 19,2\% de las publicaciones en cada caso (ver tabla 6). 
Las revistas científico-técnicas españolas de ciencias de la actividad física y el deporte: adecuación a las normas ISO y grado de normalización

Como puede observarse, la aparición conjunta de los distintos indicadores de la leyenda de cada página resulta difícil encontrarlo en las revistas del campo, ya que la única revista que más se acerca al cumplimiento completo es la 'Revista Internacional de Medicina y Ciencias de la Actividad Física y el Deporte' que, por otra parte, no posee paginación ni volumen por tratarse de una revista online. Algunas publicaciones presentan pequeñas salvedades en cuanto al cumplimiento de esta exigencia. Por ejemplo, la 'Revista de Psicología del Deporte' no cumple estrictamente con este requisito porque las páginas destinadas a la sección de librosreseñas no presenta los mencionados indicadores de la leyenda, la 'Revista de Entrenamiento Deportivo' deja de ofrecer únicamente la fecha e 'Infocoes' sólo el título del artículo.

Por otra parte, varias revistas parecen presentar formas diferentes de recoger estos datos identificativos. Así, 'Archivos de Medicina del Deporte', 'Avances en Traumatología, Rehabilitación, Cirugía, Medicina Preventiva y Deportiva' y 'Biomecánica' incluyen cada dos páginas las indicaciones de la leyenda, con la excepción del nombre de la revista. 'Psicomotricidad' recoge en la primera página de cada artículo el título de la publicación, la fecha, el fascículo y el volumen y en el resto de las páginas alterna el nombre del autor y el título del artículo. La revista 'Cairon' no incluye ninguna leyenda en la primera página de cada artículo y alterna en las siguientes el nombre de los autores, la fecha, el fascículo y el nombre de la revista, por una parte, y el título del artículo, por otra.

La paginación con caracteres arábicos es un criterio que también cumplen todas las publicaciones, a excepción evidentemente de las dos revistas on line porque no tienen páginas ('Áskesis' y 'Revista Internacional de Medicina y Ciencias de la Actividad Física y el Deporte'). La paginación correlativa de los fascículos pertenecientes a un mismo volumen se realiza en el $73,1 \%$ de las revistas y sólo el 19,2\% numera aparte las páginas de publicidad y las editan de manera que sean fácilmente separables del resto de contenidos de la publicación como exige la norma ('Biomecánica', 'Habilidad Motriz', 'Infocoes', 'Motricidad' y 'Revista de Entrenamiento Deportivo') (ver tabla 7).
El análisis de los criterios relativos al sumario de los fascículos indica que el $50 \%$ de las publicaciones no colocan dicho sumario en la primera página tras la segunda de cubierta. El título completo de los artículos aparece en el sumario del 96,2\% de las revistas, es decir, todas menos 'Áskesis'. La única revista que muestra la página de comienzo y finalización de cada artículo es 'Infocoes', a excepción lógica de las publicaciones on line que no tienen paginación y, por tanto, escapan a esta exigencia. El cumplimiento de la inclusión del autor o autores de los artículos en el sumario se produce en el $84,6 \%$ de las revistas y lo incumplen el 15,4\%, esto es, las revistas 'Áskesis', 'Infocoes', 'Revista de Biomecánica' y 'Revista Española de Educación Física y Deportes' (ver tabla 8).

En cuanto a la especificación de secciones, el 50\% de las revistas las reflejan en el sumario a continuación de los artículos y, del resto de las revistas, el 30,8\% no tiene secciones y el 19,2\% no cumple con la presentación de dichas secciones a continuación de los artículos. Sólo cinco revistas (el 19,2\%) separa las secciones con una interlínea, tal y como indica la normativa.

\section{Presentación de artículos en publicaciones periódicas (ISO 215-1986)}

El análisis de las revistas indica que el título de los artículos 'siempre' destaca en todas las publicaciones periódicas examinadas, en cambio la identificación de los autores no siempre se produce conforme a la norma objeto de análisis que exige la inclusión del nombre completo (ver tabla 9, a seguir). Si bien un porcentaje muy elevado de publicaciones, el 84,6\%, 'siempre' o 'casi siempre' identifica a los autores de los artículos, la revista 'Avances en Traumatología, Cirugía, Rehabilitación, Medicina Preventiva y Deportiva' 'nunca' referencia como 
debiera a los autores, mientras que la 'Revista de Biomecánica', 'NSW' y 'Revista Española de Educación Física y Deporte' (el 11,5\%) 'casi nunca' lo hacen conforme a la norma.

En cuanto a los resúmenes de los artículos, el 69,2\% de las revistas los incluye 'siempre' o 'casi siempre', mientras que el $11,5 \%$, es decir, las revistas 'Habilidad Motriz', 'Psicomotricidad' y 'Revista Jurídica del Deporte', 'nunca' incluyen un resumen de los artículos en el idioma de la revista. Las publicaciones que 'nunca' o 'casi nunca' presentan resúmenes de sus artículos en un segundo idioma, ya sea inglés o francés como indica la norma, son el $61,5 \%$, mientras que 'siempre' aparecen dichos resúmenes en los artículos del 19,2\% de las revistas: 'Apunts. Educación Física y Deportes', 'Motricidad', 'Revista de Biomecánica', 'Selección' y 'Tándem'. Y los mismos porcentajes se observan en la presentación de las palabras clave en cada uno de los artículos de las revistas, es decir, el $61,5 \%$ de las publicaciones 'nunca' o 'casi nunca' las presentan y el $19,2 \%$ 'siempre' incluyen palabras clave en sus artículos: 'Apunts. Educación Física y Deportes', 'Avances', 'Biomecánica', 'Motricidad' y 'Selección'. Para finalizar este punto, sólo tres revistas, 'Apunts. Educación Física y Deportes', 'Motricidad' y 'Selección', cumplen 'siempre' con todos resúmenes (en el idioma de la revista y en inglés o francés) y las palabras clave (el 11,5\%).

Al ocuparnos de la bibliografía del final de los artículos originales, se observa que 'siempre' aparece en el 73,1\% de las revistas y 'casi siempre' en un 19,2\%. Por el contrario, 'nunca' lo indica la 'Revista de Biomecánica' y 'casi nunca' la de 'Fútbol. Cuadernos Técnicos'. Los

TABLA 9

TABLA 10

TABLA 11

\section{Índice}

\begin{tabular}{|c|c|c|c|c|c|c|c|c|}
\cline { 2 - 9 } \multicolumn{1}{c|}{} & \multicolumn{4}{c|}{ Titulo destacado } & \multicolumn{4}{c|}{ Identificación autores } \\
\cline { 2 - 9 } \multicolumn{1}{c|}{} & Nunca & $\begin{array}{c}\text { Casi } \\
\text { nunca }\end{array}$ & $\begin{array}{c}\text { Casi } \\
\text { siempre }\end{array}$ & Siempre & Nunca & $\begin{array}{c}\text { Casi } \\
\text { nunca }\end{array}$ & $\begin{array}{c}\text { Casi } \\
\text { siempre }\end{array}$ & Siempre \\
\hline No & 0 & 0 & 0 & 26 & 1 & 3 & 13 & 9 \\
\hline$\%$ & 0 & 0 & 0 & 100 & 3,8 & 11,5 & 50 & 34,6 \\
\hline
\end{tabular}

Resúmenes y palabras clave

\begin{tabular}{|c|c|c|c|c|c|c|c|c|c|c|c|c|}
\cline { 2 - 14 } \multicolumn{1}{|c|}{} & \multicolumn{4}{|c|}{ Idioma de la revista } & \multicolumn{4}{c|}{ Inglés o francés } & \multicolumn{4}{c|}{ Palabras clave } \\
\cline { 2 - 14 } & Nunca & $\begin{array}{c}\text { Casi } \\
\text { nunca }\end{array}$ & $\begin{array}{c}\text { Casi } \\
\text { siemp }\end{array}$ & Siemp & Nunca & $\begin{array}{c}\text { Casi } \\
\text { nunca }\end{array}$ & $\begin{array}{c}\text { Casi } \\
\text { siemp }\end{array}$ & Siemp & Nunca & $\begin{array}{c}\text { Casi } \\
\text { nunca }\end{array}$ & $\begin{array}{c}\text { Casi } \\
\text { siemp }\end{array}$ & Siemp. \\
\hline No & 3 & 5 & 9 & 9 & 15 & 1 & 5 & 5 & 13 & 3 & 5 & 5 \\
\hline$\%$ & 11,5 & 19,2 & 34,6 & 34,6 & 57,7 & 3,8 & 19,2 & 19,2 & 50 & 11,5 & 19,2 & 19,2 \\
\hline
\end{tabular}

Texto principal: bibliografía

\begin{tabular}{|c|c|c|c|c|c|c|c|c|c|}
\cline { 2 - 10 } \multicolumn{1}{c|}{} & \multicolumn{4}{|c|}{ Artículos } & \multicolumn{4}{c|}{ Opinión } \\
\cline { 2 - 10 } & Nunca & $\begin{array}{c}\text { Casi } \\
\text { nunca }\end{array}$ & $\begin{array}{c}\text { Casi } \\
\text { siempre }\end{array}$ & Siempre & Nunca & $\begin{array}{c}\text { Casi } \\
\text { nunca }\end{array}$ & $\begin{array}{c}\text { Casi } \\
\text { siempre }\end{array}$ & Siempre & No tiene \\
\hline No & 1 & 1 & 5 & 19 & 8 & 0 & 1 & 0 & 17 \\
\hline$\%$ & 3,8 & 3,8 & 19,2 & 73,1 & 30,8 & 0 & 3,8 & 0 & 65,4 \\
\hline
\end{tabular}

\section{GRÁFICO 1}

Índices de Normalización (Comparación)

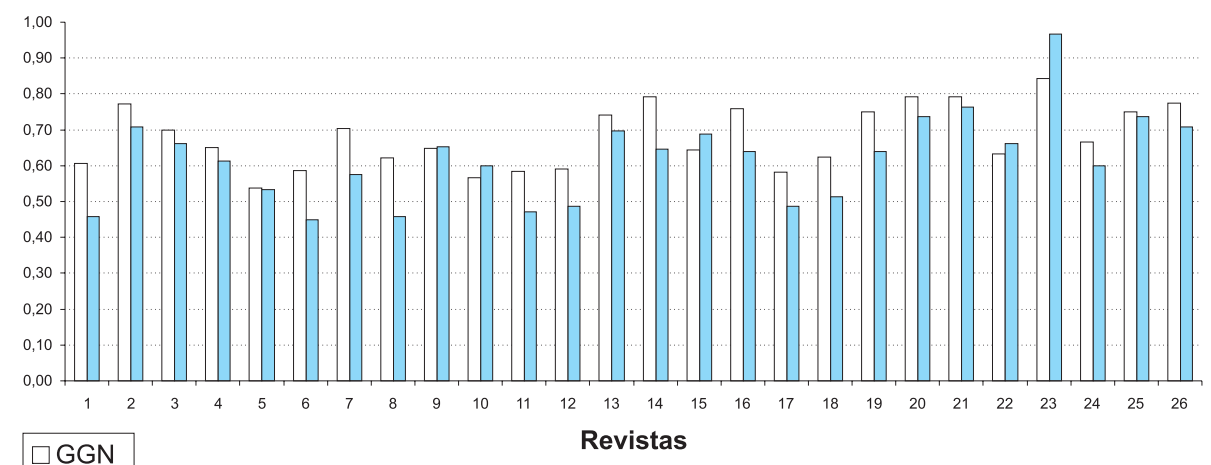

Revistas

artículos de opinión no existen en el 65,4\% de las revistas examinadas y del resto, 'casi siempre' aparece bibliografía final en el caso de la 'Revista de Psicología del Deporte'.

\section{Índices y escalas de normalización}

La mayoría de las revistas, el 76,9\%, presenta un GFN inferior a su respectivo GGN, tal y como se observa en el gráfico 1 en el que se comparan ambo índices de normalización. En la revista 'Comunicaciones Técnicas' los dos índices son idénticos y sólo en los casos de 'Espacio y Tiempo', 'NSW', 'Revista Española de Educación Física y Deportes' y 'Revista Internacional de 
Las revistas científico-técnicas españolas de ciencias de la actividad física y el deporte: adecuación a las normas ISO y grado de normalización

Medicina y Ciencias de la Actividad Física y el Deporte', el GFN es superior al GGN. Sin embargo, a pesar de esta evidencia y a excepción del último título, el GFN no se sitúa en ningún caso por encima de 0.69 , de manera que estas revistas no alcanzan niveles elevados de normalización.

En el ranking de revistas, elaborado para los dos índices de normalización (ver tabla 12), se observa que las revistas que se encuentran en los primeros lugares presentan un GFN igual o superior al GGN.

Como excepciones más destacadas está 'Motricidad' que se encuentra la segunda en el ranking del GGN y en la duodécima posición del ranking del GFN. Algo similar le ocurre a la revista 'Perspectivas de la Actividad Física y el Deporte' que de la séptima posición del GGN pasa a la treceava del GFN. También se aprecia una posición avanzada de aquellas otras que no tienen un GFN excesivamente inferior a su GGN y situado todavía en valores de 0.65 o superiores.

Finalmente, el análisis de las escalas de normalización en cuatro niveles (Muy Elevado: 0,85-1; Elevado: 0,700,84; Medio: 0,50-0,69; y Bajo: <0,50) indican que no se observan revistas con niveles 'Bajo' o 'Muy Elevado' para el GGN. Todas las revistas se reparten entre el nivel 'Elevado' y 'Medio', curiosamente casi en una misma proporción, 12 para el primero y 14 para el segundo. Por el contrario, para el GFN ya se distinguen los cuatro niveles posibles. Sólo la 'Revista Internacional de Medicina y Ciencias de la Actividad Física y el Deporte' presenta un nivel de normalización 'Muy Elevado'. En el otro extremo, se sitúan 6 revistas, el 23,1\% de los títulos, que presentan un 'Bajo' GFN. El resto de revistas se distribuyen en los niveles intermedios, situándose 6 revistas en el nivel 'Elevado' y 13 (el 50\%) en el nivel 'Medio' de GFN.

\section{DISCUSIÓN}

Para un campo con una corta tradición científica en España como el de las Ciencias de la Actividad Física y el Deporte, este primer estudio sobre la normalización de las revistas científico-técnicas describe cuál es la situación formal de los principales órganos de comunicación del conocimiento certificado del mencionado campo. A partir de esta información básica se contribuye a mejorar la calidad global de las revistas, pese a ser sólo un indicador indirecto que generalmente se combina con otros de contenido científico y difusión, pero que suele ir paralelo a la calidad intrínseca de las publicaciones periódicas o, al menos, la normalización se convierte en un prerrequisito para dicha calidad (Pérez, 2001). Además, en el marco de la comunicación científica, disponer de unos medios para la transferencia de la información que se publiquen conforme a unas normas establecidas, facilita el trabajo de todos los que producen, tratan y usan las revistas científicas (autores, editores, bibliotecarios y lectores) (Delgado, 1997). Esto, por otra parte, aumenta las posibilidades de difusión de las publicaciones, pues el cumplimiento de las normas correspondientes es uno de los primeros requisitos que se exige a una revista para su incorporación a los grandes circuitos de difusión. 
La comparación de nuestros resultados con los de otros campos de estudio españoles permite comprender mejor la situación en que se encuentran las revistas de Ciencias de la Actividad Física y el Deporte dentro del contexto español de las publicaciones científicas, aunque debe realizarse con mucha cautela debido a diferencias metodológicas en la obtención de los datos. Así, por ejemplo, los resultados de nuestro estudio son similares a los obtenidos en los campos de Tecnología, Ingeniería Mecánica y Metalúrgica (García y Faura, 1998) y de Economía (Giménez, Román y Sánchez, 1999), en lo que se refiere a la 'Presentación de las publicaciones periódicas' (norma ISO 8-1977). Sólo en dos datos del 'Título de cada página', el porcentaje de revistas del campo de Ciencias de la Actividad Física y el Deporte que los incluye es claramente inferior al de las revistas de Economía. En este sentido, el título del artículo y el nombre de los autores es recogido por sólo el 19,2\% de las publicaciones del campo, frente al 75\% de las revistas de Tecnología e Ingeniería Mecánica y Metalúrgica y el 58,3\% de las publicaciones periódicas de Economía.

No ocurre lo mismo en lo que se refiere a la 'Presentación de artículos en publicaciones periódicas' (norma ISO 215-1986). En este caso, el cumplimiento de la norma por parte de las publicaciones del campo de Ciencias de la Actividad Física y el Deporte es sustancialmente menor que en los campos de Economía y de Tecnología, Ingeniería Mecánica y Metalúrgica Los únicos aspectos en que los porcentajes de cumplimiento de las revistas de Ciencias de la Actividad Física y el Deporte son superiores a los porcentajes de las revistas de Tecnología (no de Economía), son los correspondientes a las palabras clave (el $19,2 \%$ frente al $12,3 \%$ ) y a la bibliografía final de los artículos (el 73,1\% de los casos frente al 50\%).

De esta manera, si a partir de los resultados obtenidos eligiéramos al azar una revista española científico-técnica del campo estudiado, dicha publicación tendría casi con toda seguridad un título acorde a la temática de la que se ocupara y, sin tener en cuenta el subtítulo, sería corto y fácil de citar. En su portada figuraría ese título junto con el volumen, el fascículo y la fecha. Sin embargo, es poco probable que en la portada apareciera reflejado el ISSN. Su sumario recogería muy probablemente el título completo de los artículos y el nombre de los autores, pero no expresaría para cada artículo las páginas que éste comprendiera. En este sumario tampoco se separarían con una interlínea los artículos originales de otras posibles secciones. La paginación de esta revista estaría realizada mediante números arábigos con toda seguridad, pero no podríamos esperar lo mismo de la correcta paginación de las páginas publicitarias, si tuviera. Muy probablemente, estas páginas no se habrían numerado aparte ni se habrían editado de manera que fueran fácilmente separables del resto de la revista. Del conjunto de datos de la leyenda de cada página, el número de página sería el único que encontraríamos con toda probabilidad. Si nos dispusiéramos a leer sus artículos podríamos apreciar siempre de manera destacada los títulos de los mismos y sus autores estarían correctamente identificados con bastante probabilidad. Sin embargo, sería más difícil encontrar junto a esos artículos un resumen en inglés o francés o las palabras clave. La bibliografía de estos artículos aparecería referenciada, pero no la de los artículos de opinión, en el caso de que existieran estos últimos.

Por último, los resultados obtenidos también muestran que la calidad formal del conjunto de revistas analizado es superior en aquellos aspectos que no exigen un conocimiento exhaustivo de la normativa española relativa a las publicaciones periódicas. Por el contrario, esto no ocurre así en aquellos aspectos en los que la norma hace referencia a características concretas y específicas de este tipo de publicaciones que, para su cumplimiento, requieren de una lectura detallada de la normativa a la que nos estamos refiriendo. Así parecen indicarlo los índices del GGN y GFN, ya que los criterios o indicadores fundamentales del GFN, los que marcan la diferencia de calidad formal en las revistas, se cumplen en menor medida que los generales del GGN. Además, sólo una revista se encuentra en el nivel superior de la escala de normalización del GFN ('Muy Elevado') y otras seis en el nivel siguiente ('Elevado') de dicha escala.

\section{CONCLUSIONES}

De los resultados presentados y discutidos en este trabajo se desprenden las siguientes conclusiones:

- La calidad formal relativa a la 'Presentación de las publicaciones periódicas' (norma ISO 8-1977) de las revistas científico-técnicas españolas de Ciencias de la Actividad Física y el Deporte, en especial, la correspondiente al título, la presentación, la paginación y el sumario, es comparable a la de otros campos en España (por ejemplo, el área de Economía y de Tecnología, Ingeniería Mecánica y Metalúrgica). Aun así, deben mejorarse algunas cuestiones, sobre todo, la inclusión del ISSN en las portadas de las revistas y el título del artículo y el nombre de los autores en la leyenda de cada página.

- La calidad formal de las revistas en la 'Presentación de artículos en publicaciones periódicas' (ISO 215-1986) es, en general, sustancialmente menor que la encontrada 
Las revistas científico-técnicas españolas de ciencias de la actividad física y el deporte: adecuación a las normas ISO y grado de normalización

en los campos de Economía y de Tecnología, Ingeniería Mecánica y Metalúrgica. En consecuencia, las revistas del campo deben perseguir un mayor cumplimiento de esta norma, sobre todo, en lo que se refiere a la inclusión junto a sus artículos de palabras clave y de un resumen en inglés o francés.

- Los índices de normalización creados para comparar los resultados de las distintas revistas del campo estudiado señalan que la mayoría de las revistas presentan un GFN inferior a sus respectivos GGN y que las revistas con mayor grado de normalización poseen un GFN superior o ligeramente inferior a su GGN. Esto indica que debe atenderse especialmente a los criterios o indicadores fundamentales de entre todos los relativos a la calidad formal de las revistas del campo.

- La escala de normalización del GGN divide las revistas, casi al 50\%, entre el segundo nivel de normalización considerado 'Elevado' $(0,70-0,84)$ y el tercer nivel considerado 'Medio' (0,50-0,69). La escala de normalización del GFN discrimina mucho más y marca diferencias al situar una sólo revista en el nivel 'Muy Elevado' $(0,85-1)$ de normalización, seis revistas en el nivel 'Elevado', trece en el nivel 'Medio' y las seis restantes en el nivel de normalización 'Bajo' $(<0,50)$. Los resultados de las escalas confirman lo apuntado en los índices, es decir, que los criterios fundamentales discriminan mejor el nivel de normalización de las revistas, habiendo 19 revistas en los niveles 'Medio' y 'Bajo' de la escala GFN.

Las conclusiones de nuestro estudio indican que existe un margen considerable de mejora en la normalización de las revistas científico-técnicas españolas de Ciencias de la Actividad Física y el Deporte. La normalización debería constituir, pues, una de las primeras preocupaciones de los distintos agentes de la comunidad científica para alcanzar unos estándares de calidad aceptables en las revistas, y muy especialmente de los responsables de dichas publicaciones periódicas. Además, mantener cierto grado de calidad normativa o formal no es una cuestión que, en principio, exija grandes esfuerzos a editores y directores de revistas, al menos en comparación con los que puede suponerles la mejora de la calidad de contenido y de la difusión.

Artigo recebido em 18-02-2004 e aceito para publicação em 24-04-2004.

\section{Agradecimientos}

Los resultados de este artículo derivan de un proyecto financiado por la Subdirección de Estudios y Análisis del Ministerio de Educación, Cultura y Deporte de España, convocatoria de 2001 (EA-7199).

\section{REFERENCIAS}

CALlON, M.; COURTIAL, J. P.; PENAN, H. Cienciometría. el estudio cuantitativo de la actividad científica: de la bibliometría a la vigilancia tecnológica. Gijón : Trea, 1995.

DELGADO, E. Evaluación y aplicación de las normas de presentación de publicaciones periódicas: revisión bibliográfica. Revista Española de Documentación Científica, v. 20, n. 1, p. 39-51, 1997.

DELGADO, E.; RUIZ, R. Normalización de revistas cientificas. Bibliotec 2.1. Salamanca : Ediciones Universidad de Salamanca, 1998. Disquete.

DEVÍS, J. et al. Las revistas científico-técnicas españolas de ciencias de la actividad física y el deporte: inventario y análisis de la calidad de contenido y difusión. Revista Española de Documentación Científica, v. 26, n. 2, p. 177-190, 2003.

GARCÍA DEL TORO, M. A.; FAURA, F. Estudio de las publicaciones periódicas españolas de tecnología e ingeniería mecánica y metalúrgica. Revista Española de Documentación Científica, v. 21, n. 1, p. 24-41, 1998 .

GIMÉNEZ, E.; ROMÁN, A.; SÁNCHEZ, J. M. Aplicación de un modelo de evaluación a las revistas científico-técnicas españolas de economía: una aproximación metodológica. Revista Española de Documentación Científica, v. 23, n. 3, 309-324, 1999.

; VÁZQUEZ, M. Normalización. In: ROMÁN, A.

(Coord.). La edición de revistas científicas: guía de buenos usos. Madrid: Centro de Información y Documentación Científica, p. 17-33, 2001.

INTERNATIONAL ORGANIZATION FOR STANDARDIZATION. Documentation et information: recueil de normes ISO 1. 3. ed. Ginebra : Unesco, 1988.

KRZYZANOWSKI, R. F.; FERREIRA, M. C. G. Avaliaçao de periódicos científicos e técnicos brasileiros. Ciência da Informaçao, Brasília, v. 27, n. 2, p. 165-175, 1998.

PÉREZ ÁlVAREZ-OSSORIO, J. R. La evaluación de las revistas científicas. BiD: textos universitaris de biblioteconomia i documentación, Barcelona, n. 6, jun. 2001. Disponível em: <http:// www.ub.es/biblio/bid/06perez2.htm>. Accesso em: 6 ago. 2002.

REDERO, A. L. Análisis y evaluación de las revistas científicas universitarias de Castilla y León: un estudio de normalización documental. Salamanca : Edición Sí, 2002.

RUIZ-PÉREZ, R.; PINTO MOLINA, Maria. Directrices fundamentales para la normalización de revistas científicas. Granada : Universidad; Paris : Unesco, 1990 . 